\title{
Comparison Between Models of the Decay of Light Compound Nuclei
}

\author{
V. G. Rezende and B. V. Carlson \\ Instituto Tecnológico da Aeronáutica, Departamento de Física, \\ Praça Marechal Eduardo Gomes, 50, 12228-900, São José dos Campos, SP, Brazil
}

Received on 11 August, 2005

\begin{abstract}
The decay of the compound nucleus is traditionally calculated using one of two types of statistical models, either a sequential or a simultaneous emission one. The best known sequential emission models are the Weisskopf-Ewing one and the Hauser-Feshbach one. Both sequential emission models emit only one particle at a time. A well know simultaneous emission model is the Fermi breakup one, which takes into account the fragmentation of the compound nuclei into two, three or more residual nuclei/particles. We have compared the particle and residual nucleus distributions of the Weisskopf-Ewing and Fermi breakup models in the case in which only stable nuclei/particles are emitted. We find that the Weisskopf-Ewing results in larger yields of light particles and heavy residues than the Fermi breakup model, while the latter results in larger yields of intermediate mass fragments.
\end{abstract}

\section{INTRODUCTION}

The decay of the compound nuclei is traditionally calculated using statistical models of sequential emission [1]. The most commonly used models of this type are the Weisskopf-Ewing [2] one (which does not take angular moment conservation into account) and the Hauser-Feshbach [3] one (which does). In both models, the decay of the compound nucleus to a residual nucleus occurs through sequential particle emission, until not enough energy remains for further emission.

In light compound nuclei, the excitation energy for complete disintegration of the system is relatively low and is easily reached in reactions. A model that can take this disintegration into account is the Fermi breakup one [4]. In both the sequential emission and breakup models, the cross section is written as the product of a formation cross and a branching ratio for decay, a form characteristic of decay from a statistically equilibrated system. In this project, we compare the distribution of particles and residual nuclei that results from calculations of the Weisskopf-Ewing model of sequential emission and the Fermi breakup one.

\section{WEISSKOPF-EWING MODEL}

The Weisskopf-Ewing cross section can be written as

$$
\sigma_{a c}=\sigma_{a, a b s} \frac{Y_{c}}{\sum_{b} Y_{b}} .
$$

The emission factor $Y_{c}$ is proportional to the absorption cross section and can be written as

$$
Y_{c}\left(\varepsilon_{c}\right)=\left(2 S_{c}+1\right) \frac{2 \mu_{c}}{\pi \hbar^{2}} \varepsilon_{c} \sigma_{c, a b s}\left(\varepsilon_{c}\right),
$$

where $\mu_{c}$ is the reduced mass in channel $c$ and $\varepsilon_{c}$ is the centerof-mass energy of the channel.

The emission factor $Y_{c}$ must be modified to take into account the density of states. This factor for the WeisskopfEwing model is given by the expression

$$
Y_{c}\left(\varepsilon_{c}\right) \rightarrow Y_{c}\left(\varepsilon_{c}\right) \omega_{c}\left(E_{c}\right) d \varepsilon_{c},
$$

where $\omega_{c}\left(E_{c}^{*}\right)$ is the level density of the residual nucleus, with

$$
E_{c}^{*}=E_{c n}^{*}-\varepsilon_{c}-B_{c},
$$

and $B_{c}$ is the separation energy of the particle emitted in channel $c$.

The Weisskopf-Ewing cross section can thus be written as

$$
\begin{aligned}
& \frac{d \sigma_{a c}}{d \varepsilon_{c}}=\sigma_{a, a b s} \\
& \times \frac{\left(2 S_{c}+1\right) 2 \mu_{c} \sigma_{c, a b s}\left(\varepsilon_{c}\right) \omega_{c}\left(E_{c}^{*}\right)}{\sum_{b} \int_{0}^{E_{x}^{*}} d \varepsilon_{b}\left(2 S_{b}+1\right) 2 \mu_{b} \sigma_{b, a b s}\left(\varepsilon_{b}\right) \omega_{b}\left(E_{b}^{*}\right)} .
\end{aligned}
$$

In the calculations using the model, we use a global fit to the absorption cross sections, Gilbert-Cameron level densities and a level density parameter of $a=A / 7.1(\mathrm{MeV})^{-1}$. We consider the emission of particle-bound nuclei of mass $A<7$. Ground states masses are taken from the 1998 mass table of AudiWapstra.

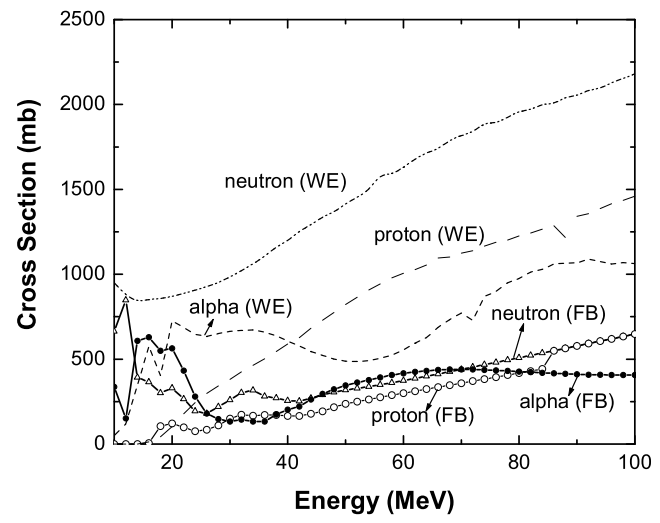

FIG. 1: Weisskopf-Ewing and Fermi breakup production cross sections (in millibarns) of neutrons, protons and alphas as a function of the initial excitation energy. 


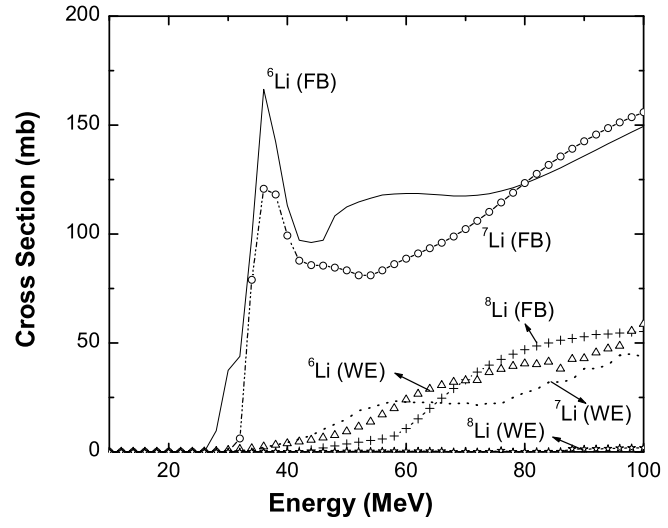

FIG. 2: Weisskopf-Ewing and Fermi breakup production cross sections (in millibarns) of several intermediate mass fragments as a function of the initial excitation energy.

\section{FERMI BREAKUP MODEL}

Here, the total kinetic energy $E$ for a breakup into $n$ fragments is calculated using the expression

$$
E=U+M(A, Z)-E_{C o u l}-\sum_{b=1}^{n}\left(m_{b}+\varepsilon_{b}\right),
$$

where $m_{b}$ and $\varepsilon_{b}$ are the masses and excitation energies of the fragments and $E_{C o u l}$ is the Coulomb barrier for the channel, given by

$$
E_{\text {Coul }}=\frac{3 e^{2}}{5 r_{0}}\left(1+\frac{V}{V_{0}}\right)^{-1 / 3}\left(\frac{Z^{2}}{A^{\frac{1}{3}}}-\sum_{b=1}^{n} \frac{Z^{2}}{A_{b}^{\frac{1}{3}}}\right),
$$

where $V / V_{0}=1$ is normally used.

The Fermi breakup cross section takes the form

$$
\sigma_{a c}=\sigma_{a, a b s} \frac{w_{c}}{\sum_{b} w_{b}}
$$

where $w_{c}$ is the emission rate from channel $c$.

The total probability for separation in $n$ components in channel $b$ is given by the expression

$$
w_{b}(E)=\left(\frac{V}{\Omega}\right)^{n-1} \rho_{b}(E)
$$

where $V$ is the decay volume of the system and $\Omega=(2 \pi \hbar)^{3}$ is the normalization volume of the system.

The density of final states $\rho_{b}(E)$ can be defined as the product of three factors,

$$
\rho_{b}(E)=M_{n}(E) S_{n} G_{n},
$$

where the first term is the phase space factor, defined as,

$$
\begin{aligned}
M_{n}(E)= & \int_{-\infty}^{+\infty} \ldots \int_{-\infty}^{+\infty} \delta\left(\sum_{b_{j}=1}^{n} \vec{p}_{b_{j}}\right) \\
& \times \delta\left(\sum_{b_{j}=1}^{n} \frac{p_{b_{j}}^{2}}{2 m_{b_{j}}}-E\right) \prod_{b_{j}=1}^{n} d^{3} p_{b_{j}},
\end{aligned}
$$

where $\vec{p}_{b_{j}}$ is the momentum of fragment $b_{j}$.

The second term is the spin factor determined by the expression

$$
S_{n}=\prod_{b_{j}=1}\left(2 S_{b_{j}}+1\right)
$$

which furnishes the number of states with different spin orientations.

The last term is the permutation factor given by

$$
G_{n}=\prod_{b_{j}=1}^{k} \frac{1}{n_{b_{j}} !}
$$

which takes into account the identity of the fragments in the final state, where $n_{b_{j}}$ is the number of particles of type $b_{j}$. In the calculations shown, we include all particle-bound states of the decay products and also consider the nucleus ${ }^{8} \mathrm{Be}$, due to its long lifetime. Ground states masses are taken from the 1998 mass table of Audi-Wapstra.

\section{CONCLUSIONS}

Overall, as shown in Figs. 1 and 2, we find that the WeisskopfEwing calculations result in the production of more light particles and heavy residues, while the Fermi breakup calculations yield more intermediate mass residues. This is probably due to the fact that the emisson of particle-unbound nuclei has not been considered in the Fermi breakup calculations. Such emissions are observed experimentally. We plan to include them in the future.
[1] E. Fermi, Prog. Theor. Phys. 5, 570 (1950).

[2] B. V. Carlson, A Brief Overview of Models of Nucleon- Induced Reactions, ICTP Lecture Notes 12, 181 (2002).

[3] W. Hauser and H. Fesbach, Phys. Rev. 87, 366 (1952).
[4] A. S. Botvina, A. S. Iljinov, I. N. Mishustin, J. P. Bondorf, R. Donangelo, and Sneppen, Nucl. Phys. A 475, 663 (1987). 